\title{
Impact of Provider Volume on Outcomes of Patients With Hodgkin Lymphoma
}

\author{
Catherine Ireland ${ }^{\mathrm{a}}$, Eric Wiedower ${ }^{\mathrm{b}, \mathrm{c}}$, Muhammad Mirza $^{\mathrm{b}}$, Melissa Crawley ${ }^{\mathrm{b}}$, \\ Alexandria Tran ${ }^{\mathrm{a}}$, George Yaghmour ${ }^{\mathrm{b}}$, Mike G. Martin ${ }^{\mathrm{b}}$
}

\begin{abstract}
Background: While the provider volume-outcome relationship has been established for many complex surgeries and invasive procedures, the provider volume impact on outcomes for Hodgkin lymphoma (HL) is less certain. We hypothesized that high-volume providers (HVPs) may have superior outcomes compared with low-volume providers (LVPs).
\end{abstract}

Methods: We performed a chart-based, retrospective review of all patients receiving adriamycin, doxorubicin, bleomycin, vinblastine, and dacarbazine (ABVD) for HL at the West Cancer Center from January 2010 to June 2015. Patients were divided into HVP ( $>3$ inpatient chemotherapy $(\mathrm{CT})$ /month $(\mathrm{m}))$ versus LVP $(<3 \mathrm{CT}$ per $\mathrm{m})$ groups. Of 95 patients identified, 93 received at least one dose of ABVD, 21 treated by HVP and 72 by LVP. Patient characteristics were well balanced between groups.

Results: HVPs were less likely to prescribe dose delays (odds ratio (OR): 0.32; confidence interval (CI): $0.16-0.65 ; \mathrm{P}=0.0007)$ and to hold doses for afebrile neutropenia (OR: 0.05; CI: $0.00-0.85$; P = $0.0006)$. HVP delivered significantly fewer prophylactic growth factors $(0 \%$ of doses vs. $42 \%$, OR: $0.00 ; \mathrm{CI}<0.00-0.06 ; \mathrm{P}<0.0001)$. Both event-free survival (EFS) (HR: 6.68; CI: $1.10-7.63 ; \mathrm{P}=0.0321$ ) and overall survival (OS) (HR: 3.68; CI: $1.11-12.22 ; \mathrm{P}=0.032)$ were significantly inferior in the patients treated by LVP.

Conclusions: In this study, patients with HL treated by LVP had inferior outcomes compared with those treated by HVP. HVPs were less likely to prescribe dose delays, hold doses for afebrile neutropenia or administer growth factor prophylaxis. These observations need to be confirmed in alternative datasets.

Keywords: Hodgkin; Lymphoma; Volume

Manuscript submitted March 7, 2018, accepted April 11, 2018

aDepartment of Internal Medicine, The University of Tennessee Health Science Center, Memphis, TN 38138, USA

${ }^{b}$ Department of Hematology/Oncology, The West Cancer Center, The University of Tennessee Health Science Center, Memphis, TN 38138, USA

${ }^{\mathrm{c} C o r r e s p o n d i n g ~ A u t h o r: ~ E r i c ~ R . ~ W i e d o w e r, ~ D e p a r t m e n t ~ o f ~ H e m a t o l o g y / O n-~}$ cology, West Cancer Center, 7945 Wolf River Blvd, Memphis, TN 38138, USA.Email: ewiedower@westclinic.com

doi: https://doi.org/10.14740/wjon1093w

\section{Introduction}

While the relationship between hospital and provider volume and outcomes has been well established for complex surgeries and invasive procedures, the impact of volume on outcomes for patients with hematologic malignancies is less certain [1$3]$. We previously reported that low-volume centers have higher inpatient mortality (odds ratio (OR): $3.26 ; \mathrm{P}<0.001$ ) than high-volume centers when treating acute myeloid leukemia (AML) [4]. High-volume centers in this study were defined as $>75$ th percentile, which translated into $>3$ inpatient chemotherapy (CT)/month (m), versus the other centers.

Similar to AML, the treatment of Hodgkin lymphoma (HL) is nuanced and depends on dose intensity; therefore, outcomes may be affected by provider experience and guideline adherence [5-7]. For example, the National Comprehensive Cancer Network (NCCN) guidelines state that leukopenia is not an indication for dose reductions or delay of chemotherapy with adriamycin, bleomycin, vinblastine, and dacarbazine (ABVD) as febrile neutropenia is rare [8-10]. Similarly, the routine use of myeloid growth factors is not recommended and may be associated with increased risk of bleomycin pulmonary toxicity [11]. Failure to follow these paradigms that are counter-intuitive to the use of chemotherapy in other malignancies may lead to inferior outcomes.

We hypothesized that an outcome-volume relationship would also exist for high-volume providers (HVPs) versus low-volume providers (LVPs) caring for patients with HL.

\section{Patients and Methods}

This was a retrospective study approved by the Institutional Review Board at the University of Tennessee. The patients were identified through a pharmacy query of the electronic medical records for whom ABVD was prescribed at the West Cancer Center from January 2010 to June 2015. Patients with a diagnosis of $\mathrm{HL}$ having received at least one dose of ABVD were included in the analysis. Patients were divided into two groups according to the primary provider volume, HVP (provider prescribing $>3 \mathrm{CT}$ per $\mathrm{m})$ and LVP $(<3 \mathrm{CT}$ per $\mathrm{m})$ groups based on previous studies [4]. Group characteristics were compared using Fisher's exact test. Event-free survival (EFS) and overall survival (OS), as defined by treatment failure or death from any cause and death from any cause, respectively, were com- 
Table 1. Baseline Characteristics of the HVP and LVP Cohorts

\begin{tabular}{|clll}
\hline & HVP & LVP & P-value \\
\hline Age (range) & $38.1(23-69)$ & $43.4(19-88)$ & 0.224 \\
Percent white & 72 & 52 & \\
Percent males & 56 & 29 & \\
IPS score & & & \\
0 & $14 \%$ & $13 \%$ & \\
1 & $33 \%$ & $38 \%$ & \\
\hline 2 & $23 \%$ & $17 \%$ & \\
\hline 3 & $14 \%$ & $13 \%$ & \\
\hline 4 & $10 \%$ & $9 \%$ & \\
\hline 5 & 0 & $4 \%$ & \\
\hline Unknown & $5 \%$ & $7 \%$ & \\
Stage & & $8 \%$ & \\
\hline 1A & $5 \%$ & $35 \%$ & \\
\hline 2A & $33 \%$ & $15 \%$ & \\
\hline 3A & $0 \%$ & $8 \%$ & \\
\hline 3B & $5 \%$ & $10 \%$ & \\
\hline 4A & $19 \%$ & & \\
\hline 4B & $14 \%$ & & \\
\hline & & & \\
\hline
\end{tabular}

pared using Mantel-Cox test. All analyses were performed using Microsoft Excel 2011 v14.5.7 and GraphPad Prism v6.0f. $\mathrm{P}$-values $<0.05$ were considered statistically significant and all $\mathrm{P}$-values were two-sided.

\section{Results}

Ninety-five patients were identified in the database and 93 patients received at least one dose of ABVD. One HVP cared for 21 patients in the HPV group, while 15 LVP cared for the 72 patients in the LVP group (range 1 - 9 patients per provider) delivering a mean of 3.3 and 0.8 doses of $\mathrm{CT}$ per month, respectively. While there were more females in the HVP cohort versus LVP (56\% vs. 29\%), there were no statistical differences in age, international prognostic score, which includes gender, or stage distribution between the HVP and LVP groups (Table 1). Patients with unfavorable risk ( $\geq 2 \mathrm{~B}$ ) were also balanced between the groups (HVP 62\% vs. LVP 57\%, P = 0.803). Median follow-up was $564(106$ - 1,832) days for HVP versus 582 (14 - 1,844) for LVP, with no patients lost to follow-up.

Total dose delays were less likely performed by HVP (OR: 0.32; CI: $0.15-0.65 ; \mathrm{P}=0.0007)$, and HVP was less likely to hold treatment for afebrile neutropenia (OR: 0.05; CI: 0.00 $0.85 ; \mathrm{P}=0.0006)$. The toxic death rate was not statistically lower for patients in the HVP group (OR: 0.18; CI: 0.010 3.189; $\mathrm{P}=0.1913)$. HVP delivered significantly fewer prophylactic growth factors ( $0 \%$ vs. 42\%; OR: 0.00 ; CI $<0.00-0.06$; $\mathrm{P}<0.0001)$. Both EFS (HR: 6.68; CI: $1.10-7.62 ; \mathrm{P}=0.0321$ ) and OS (HR: 3.68; CI: 1.11 - 12.22; P = 0.032) were significantly inferior in the LVP group (Fig. 1).

\section{Discussion}

In a previous study, we investigated the outcome-volume relationship of AML which showed a difference in mortality between high- and low-volume centers [4]. Similarly, the results of this study show a correlation between outcomes and provider volume in the treatment of HL at our cancer center. This may be explained in part by lack of familiarity with the NCCN guidelines that lead to treatment delays for afebrile neutropenia and consequently lower dose-intensity.

The overall cure rate of HL approaches $90 \%$ with appropriate treatment [12]. Davis et al demonstrated that mortality rates in patients with HL treated in the community were 1.5 times higher than those treated at National Cancer Institute designated cancer centers [13]. For a highly curable disease, prevention of treatment-related complications and mortality is a major concern. Febrile neutropenia, bleomycin toxicity, and poor dose-intensity during treatment are major therapy-related factors affecting outcomes in HL [8-11]. These complications can be minimized by strict adherence to current treatment guidelines. Mauch et al described that deaths from HL decreased with time from treatment but the excess risk of death from all causes remained constant with time from treatment and was $1.2 \%$ per year over the first 20 years. Age at diagnosis (age $\geq 40$ ) and combined CT/radiotherapy were significant risk factors for all cause death [14].

The outcome-volume relationship was hypothesized when Luft et al demonstrated a relationship between surgical volume and patient outcome [15]. Accordingly, several studies have supported a relationship between hospital volume and shortterm outcomes for malignancies treated for high-risk surgical procedures [16]. Few studies exist investigating the outcomevolume relationship of hematologic malignancies. A retrospective study by Hillner et al hypothesized that high-volume hospital and physicians lead to better outcomes in cancer treatment, but this study mostly consisted of surgical cases [17]. Interestingly, Birkmeyer et al illustrated that relations between surgical mortality is primarily mediated by surgeon rather than hospital volume, as high-volume surgeons had better outcomes than low-volume surgeons despite being in a high-volume hospital [18]. Kimm et al compared treatment efficacy between type of treatment center and/or the center caseload; it was found that neither caseload nor center type (university hospital, non-university hospital, hematology-oncology practices) affect the progression-free survival for HL. This study implied that treatment protocols were followed even in centers with lower caseloads [19]. In contrast, our study evaluated HVP and LVP outcomes within a cancer center and found a significant difference in survival with superior outcomes in those treated by HVP.

Burel et al found that when treating diffuse large B-cell lymphoma, poor adherence to R-CHOP therapy was strongly associated with poor OS. In the study, treatment adherence was measured by the ratio between received and planned dose intensity, and was found to affect OS [20]. Examining hospital volume and adherence to guidelines, Phippen et al found that in advanced ovarian cancer, compliance to NCCN recommended treatment guidelines with optimal cytoreductive surgery in low-volume centers can have similar outcomes to high-volume centers. Patients treated in the low-volume center with adherence to NCCN 


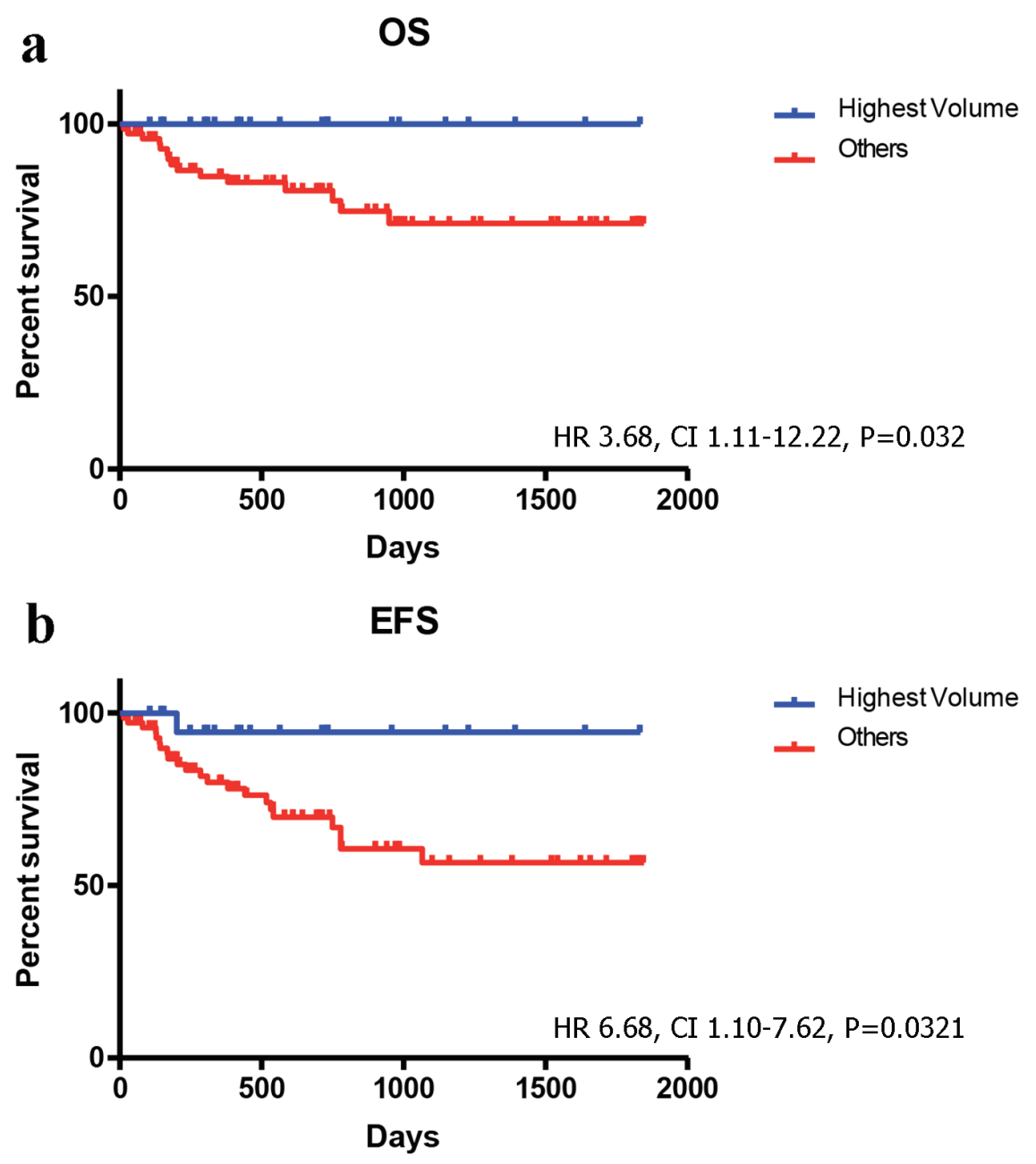

Figure 1. Survival outcomes for the HVP and LVP cohorts: (a) OS; (b) EFS.

guidelines had OS rates similar to high-volume centers [21].

Socioeconomic factors such as insurance status, residence and treatment institution also affect treatment outcomes. Loberiza et al showed that patients in rural areas are at increased risk of death when compared with patients treated at a university center or community center in urban areas [22]. Similarly, Parikh et al revealed that patients with Medicare or no insurance had worse 5-year OS rates compared with favorably insured patients as they were less likely to receive radiotherapy, start CT promptly and less commonly treated at academic or research centers [23].

Our study is the first to assess the provider-volume impact on HL with outcomes being inferior in those treated by LVP versus HVP. Larger studies are needed to further investigate these results and efforts to increase homogeneity of patient care and adherence to guidelines may improve outcomes.

\section{Author Contributions}

EW, MMi, AT, MC and GY wrote the paper. MGM developed research, analyzed results, made the figures and was senior author. CI analyzed results and wrote the paper. MMe reviewed and edited paper.

\section{Conflict of Interest}

The authors declare no conflict of interest.

\section{References}

1. Kahn JM, Goss CH, Heagerty PJ, Kramer AA, O'Brien $\mathrm{CR}$, Rubenfeld GD. Hospital volume and the outcomes of mechanical ventilation. N Engl J Med. 2006;355(1):4150 .

2. Cram P, Rosenthal GE, Vaughan-Sarrazin MS. Cardiac revascularization in specialty and general hospitals. N Engl J Med. 2005;352(14):1454-1462.

3. Birkmeyer JD, Siewers AE, Finlayson EV, Stukel TA, Lucas FL, Batista I, Welch HG, et al. Hospital volume 
and surgical mortality in the United States. N Engl J Med. 2002;346(15):1128-1137.

4. Giri S, Pathak R, Aryal MR, Karmacharya P, Bhatt VR, Martin MG. Impact of hospital volume on outcomes of patients undergoing chemotherapy for acute myeloid leukemia: a matched cohort study. Blood. 2015;125(21):33593360 .

5. Epelbaum R, Faraggi D, Ben-Arie Y, Ben-Shahar M, Haim N, Ron Y, Robinson E, et al. Survival of diffuse large cell lymphoma. A multivariate analysis including dose intensity variables. Cancer. 1990;66(6):1124-1129.

6. Lyman GH, Dale DC, Friedberg J, Crawford J, Fisher RI. Incidence and predictors of low chemotherapy dose-intensity in aggressive non-Hodgkin's lymphoma: a nationwide study. J Clin Oncol. 2004;22(21):4302-4311.

7. Lyman GH. Impact of chemotherapy dose intensity on cancer patient outcomes. J Natl Compr Canc Netw. 2009;7(1):99-108.

8. Boleti E, Mead GM. ABVD for Hodgkin's lymphoma: full-dose chemotherapy without dose reductions or growth factors. Ann Oncol. 2007;18(2):376-380.

9. Kuderer NM, Dale DC, Crawford J, Cosler LE, Lyman GH. Mortality, morbidity, and cost associated with febrile neutropenia in adult cancer patients. Cancer. 2006;106(10):2258-2266.

10. Minuk LA, Monkman K, Chin-Yee IH, Lazo-Langner A, Bhagirath V, Chin-Yee BH, Mangel JE. Treatment of Hodgkin lymphoma with adriamycin, bleomycin, vinblastine and dacarbazine without routine granulocyte-colony stimulating factor support does not increase the risk of febrile neutropenia: a prospective cohort study. Leuk Lymphoma. 2012;53(1):57-63.

11. Martin WG, Ristow KM, Habermann TM, Colgan JP, Witzig TE, Ansell SM. Bleomycin pulmonary toxicity has a negative impact on the outcome of patients with Hodgkin's lymphoma. J Clin Oncol. 2005;23(30):76147620.

12. Canellos GP, Rosenberg SA, Friedberg JW, Lister TA, Devita VT. Treatment of Hodgkin lymphoma: a 50-year perspective. J Clin Oncol. 2014;32(3):163-168.

13. Davis S, Dahlberg S, Myers MH, Chen A, Steinhorn SC. Hodgkin's disease in the United States: a comparison of patient characteristics and survival in the Centralized Cancer Patient Data System and the Surveillance, Epidemiology, and End Results Program. J Natl Cancer Inst.
1987;78(3):471-478.

14. Mauch PM, Kalish LA, Marcus KC, Shulman LN, Krill E, Tarbell NJ, Silver B, et al. Long-term survival in Hodgkin's disease relative impact of mortality, second tumors, infection, and cardiovascular disease. Cancer J Sci Am. 1995;1(1):33-42.

15. Luft HS, Bunker JP, Enthoven AC. Should operations be regionalized? The empirical relation between surgical volume and mortality. N Engl J Med. 1979;301(25):13641369.

16. Gruen RL, Pitt V, Green S, Parkhill A, Campbell D, Jolley D. The effect of provider case volume on cancer mortality: systematic review and meta-analysis. CA Cancer J Clin. 2009;59(3):192-211.

17. Hillner BE, Smith TJ, Desch CE. Hospital and physician volume or specialization and outcomes in cancer treatment: importance in quality of cancer care. J Clin Oncol. 2000;18(11):2327-2340.

18. Birkmeyer JD, Stukel TA, Siewers AE, Goodney PP, Wennberg DE, Lucas FL. Surgeon volume and operative mortality in the United States. N Engl J Med. 2003;349(22):2117-2127.

19. Klimm B, Brillant C, Skoetz N, Muller H, Engert A, Borchmann P. The effect of specialized cancer treatment centers on treatment efficacy in Hodgkin's lymphoma. Dtsch Arztebl Int. 2012;109(51-52):893-899.

20. Borel C, Lamy S, Compaci G, Recher C, Jeanneau P, Nogaro JC, Bauvin E, et al. A longitudinal study of nonmedical determinants of adherence to R-CHOP therapy for diffuse large B-cell lymphoma: implication for survival. BMC Cancer. 2015;15:288.

21. Phippen NT, Barnett JC, Lowery WJ, Miller CR, Leath CA, 3rd. Surgical outcomes and national comprehensive cancer network compliance in advanced ovarian cancer surgery in a low volume military treatment facility. Gynecol Oncol. 2013;131(1):158-162.

22. Loberiza FR, Jr., Cannon AJ, Weisenburger DD, Vose JM, Moehr MJ, Bast MA, Bierman PJ, et al. Survival disparities in patients with lymphoma according to place of residence and treatment provider: a population-based study. J Clin Oncol. 2009;27(32):5376-5382.

23. Parikh RR, Grossbard ML, Green BL, Harrison LB, Yahalom J. Disparities in survival by insurance status in patients with Hodgkin lymphoma. Cancer. 2015;121(19):35153524. 